\title{
Indistinguishability-enabled coherence for quantum metrology
}

\author{
Alessia Castellini, ${ }^{1, *}$ Rosario Lo Franco, ${ }^{1,2, \dagger}$ Ludovico Lami, ${ }^{3, \$}$ Andreas Winter, ${ }^{4, \S}$ \\ Gerardo Adesso, ${ }^{3, \|}$ and Giuseppe Compagno ${ }^{1, \pi}$ \\ ${ }^{1}$ Dipartimento di Fisica e Chimica - Emilio Segrè, Università di Palermo, via Archirafi 36, 90123 Palermo, Italy \\ ${ }^{2}$ Dipartimento di Ingegneria, Università di Palermo, Viale delle Scienze, Edificio 6, 90128 Palermo, Italy \\ ${ }^{3}$ School of Mathematical Sciences and Centre for the Mathematics and Theoretical Physics of Quantum Non-Equilibrium Systems, \\ University of Nottingham, University Park, Nottingham NG/ 2RD, United Kingdom \\ ${ }^{4}$ ICREA \& Física Teórica: Informació i Fenómens Quàntics, Departament de Física, \\ Universitat Autònoma de Barcelona, ES-08193 Bellaterra (Barcelona), Spain
}

(Received 22 March 2019; published 8 July 2019)

\begin{abstract}
Quantum coherence plays a fundamental and operational role in different areas of physics. A resource theory has been developed to characterize the coherence of distinguishable particles systems. Here we show that indistinguishability of identical particles is a source of coherence, even when they are independently prepared. In particular, under spatially local operations, states that are incoherent for distinguishable particles, can be coherent for indistinguishable particles under the same procedure. We present a phase discrimination protocol, in which we demonstrate the operational advantage of using two indistinguishable particles rather than distinguishable ones. The coherence due to the quantum indistinguishability significantly reduces the error probability of guessing the phase, using the most general measurements. The role played by particle statistics in the protocol is also investigated.
\end{abstract}

DOI: 10.1103/PhysRevA.100.012308

\section{INTRODUCTION}

Quantum coherence concerns the possibility to create a quantum state as a superposition of two or more different configurations. Recently, a resource theory of coherence has been proposed, in order to characterize, quantify, and exploit coherence [1-7]. Choosing a reference basis, diagonal, and nondiagonal states in that basis are called incoherent (free) states and coherent (resource) states, respectively. The free operations to which we have access, in accordance with physical constraints imposed on the system, are the so-called incoherent operations. They cannot create coherence starting from incoherent states. It has been shown that coherence of a quantum state has an operational relevance in the implementation of phase discrimination protocols using distinguishable particles: the robustness of coherence quantifies not only the amount of coherence of a quantum state but also the advantage offered by its presence in a metrology protocol $[3,8]$. In the context of quantum thermodynamics, a link between the coherence of a quantum state and the extractable work has been identified [9], and very recently the role of quantum coherence as a resource for the nonequilibrium entropy production has been pinpointed [10].

\footnotetext{
*alessia.castellini@unipa.it

†rosario.lofranco@unipa.it

¥ludovico.lami@gmail.com

§andreas.winter@uab.cat

"Gerardo.Adesso@ nottingham.ac.uk

Igiuseppe.compagno@unipa.it
}

The resource theory of coherence is well defined for singleparticle systems and for systems of multidistinguishable particles. On the other hand, recently, it has been shown that indistinguishability of identical particles confers on quantum systems original properties that can be used as sources of entanglement to implement quantum information processes, impossible to perform if particles are distinguishable [11-18].

In this work, we generalize the resource theory of coherence to systems of identical particles, using a particlebased approach that only allows physical labels to address the particles in the system $[19,20]$. Our aim is to show how indistinguishability may be a source of operational coherence, highlighting also the role of particle statistics. Clearly, and in contrast to the case of distinguishable particles, to achieve this we have to consider at least two (identical) particles.

\section{COHERENCE OF IDENTICAL PARTICLE STATES}

Let us consider, in the no-label formalism [19-21], two identical spins (that is, particles with a two-level internal state space) in the mixed state

$$
\rho^{\mathcal{I}}=\sum_{\sigma, \tau=\downarrow, \uparrow} p_{\sigma \tau}\left|\psi \sigma, \psi^{\prime} \tau\right\rangle\left\langle\psi \sigma, \psi^{\prime} \tau\right|,
$$

where $\mathcal{I}$ stands for identical, $\psi$ and $\psi^{\prime}$ represent the spatial degrees of freedom and $\sigma$ and $\tau$ are the pseudospins. The coefficients $p_{\sigma \tau}$ are such that $\operatorname{Tr}\left[\rho^{\mathcal{I}}\right]=1$. Each term $\left|\psi \sigma, \psi^{\prime} \tau\right\rangle$ in the sum is the state of two identical particles, one of which is characterised by $\psi$ and $\sigma$, the other by $\psi^{\prime}$ and $\tau$. This state is a global object, which crucially cannot be written as a tensor product of single-particle states, i.e., $\left|\psi \sigma, \psi^{\prime} \tau\right\rangle \neq|\psi \sigma\rangle \otimes\left|\psi^{\prime} \tau\right\rangle$ 
To define the coherence of this state, an orthonormal basis has to be chosen. Let us now make some considerations in order to opportunely choose a preferred basis, depending on the possible operations that can be performed for free on the system. A resource theory requires the identification of a physically and operationally meaningful set of free operations, which enable manipulation of resourceful states. Analogously to the case of distinguishable particles, both singleparticle and two-particle operations on the system are allowed. Identical particles are physically not addressable individually $[22,23]$, thus the concept of single-particle operation may seem ill defined, requiring us to differentiate them. As an example, since the global Hilbert space is not a tensor product of two single-spin spaces, if a CNOT (free operation for distinguishable particles [6]) has to be performed, it is not possible to differentiate between the control and the target particle.

Following the procedure to identify and quantify the useful entanglement between identical particles [18], in order to characterize the quantum coherence we here adopt a framework based on SLOCC, which consists of the identification of specific separate spatial regions to make single-particle and two-particle local measurements. In particular, we choose the basis $\mathcal{B}^{I}=\{|\mathrm{L} \sigma, \mathrm{R} \tau\rangle ; \sigma, \tau=\downarrow, \uparrow\}$, where $|\mathrm{L}\rangle$ and $|\mathrm{R}\rangle$ are two states spatially localized in the separate regions $\mathcal{L}$ and $\mathcal{R}$, respectively. If $\rho^{\mathcal{I}}$ is diagonal in the chosen basis, it is incoherent, otherwise we say that $\rho^{\mathcal{I}}$ is coherent. We point out that if $|\psi\rangle$ and $\left|\psi^{\prime}\right\rangle$ spatially overlap, the measurement regions $\mathcal{L}$ and $\mathcal{R}$ are taken within the shared spatial region.

For nonidentical particles, identified by labels $\mathrm{A}$ and $\mathrm{B}$, the state of Eq. (1) would correspond to $\rho^{\mathcal{N I}}=$ $\sum_{\sigma, \tau} p_{\sigma \tau}(|\psi \sigma\rangle\langle\psi \sigma|)_{\mathrm{A}} \otimes\left(\left|\psi^{\prime} \tau\right\rangle\left\langle\psi^{\prime} \tau\right|\right)_{\mathrm{B}}$. Particles being addressable, the framework of spatially local operations and classical communication coincides with the LOCC one and the operational basis is $\mathcal{B}=\left\{|\mathrm{L} \sigma\rangle_{\mathrm{A}} \otimes|\mathrm{R} \tau\rangle_{\mathrm{B}} ; \sigma, \tau=\downarrow, \uparrow\right\}$. Regardless of the spatial overlap of $|\psi\rangle$ and $\left|\psi^{\prime}\right\rangle, \rho^{\mathcal{N I}}$ is incoherent, in fact

$$
\begin{aligned}
\rho_{\mathrm{LR}}^{\mathcal{N} \mathcal{I}}= & \frac{1}{\mathcal{N}} \sum_{\sigma \tau} p_{\sigma \tau}\left(\Pi_{\mathrm{L}}|\psi \sigma\rangle\langle\psi \sigma| \Pi_{\mathrm{L}}\right)_{\mathrm{A}} \\
& \otimes\left(\Pi_{\mathrm{R}}\left|\psi^{\prime} \tau\right\rangle\left\langle\psi^{\prime} \tau\right| \Pi_{\mathrm{R}}\right)_{\mathrm{B}} \\
= & \sum_{\sigma \tau} p_{\sigma \tau}(|\mathrm{L} \sigma\rangle\langle\mathrm{L} \sigma|)_{\mathrm{A}} \otimes(|\mathrm{R} \tau\rangle\langle\mathrm{R} \tau|)_{\mathrm{B}}
\end{aligned}
$$

where $\Pi_{\mathrm{L}}=\sum_{\sigma}(|\mathrm{L} \sigma\rangle\langle\mathrm{L} \sigma|)_{\mathrm{A}}$ and $\Pi_{\mathrm{R}}=\sum_{\sigma}(|\mathrm{R} \sigma\rangle\langle\mathrm{R} \sigma|)_{\mathrm{B}}$. As a result, the mixed state $\rho^{\mathcal{N I}}$ for two independently prepared nonidentical particles, being diagonal in the chosen basis, is incoherent.

Projecting, on the other hand, $\rho^{\mathcal{I}}$ of Eq. (1) on the chosen subspace spanned by the basis $\mathcal{B}^{\mathcal{I}}$, we obtain the following state:

$$
\begin{aligned}
\rho_{\mathrm{LR}}^{\mathcal{I}}= & \frac{1}{\mathcal{N}} \sum_{\sigma, \tau=\downarrow, \uparrow} p_{\sigma \tau}\left(|l|^{2}\left|r^{\prime}\right|^{2}|\mathrm{~L} \sigma, \mathrm{R} \tau\rangle\langle\mathrm{L} \sigma, \mathrm{R} \tau|\right. \\
& +\eta l r^{\prime} l^{*} r^{*}|\mathrm{~L} \sigma, \mathrm{R} \tau\rangle\langle\mathrm{L} \tau, \mathrm{R} \sigma| \\
& +\eta l^{\prime} r l^{*} r^{*}|\mathrm{~L} \tau, \mathrm{R} \sigma\rangle\langle\mathrm{L} \sigma, \mathrm{R} \tau| \\
& \left.+\eta\left|l^{\prime}\right|^{2}|r|^{2}|\mathrm{~L} \tau, \mathrm{R} \sigma\rangle\langle\mathrm{L} \tau, \mathrm{R} \sigma|\right)
\end{aligned}
$$

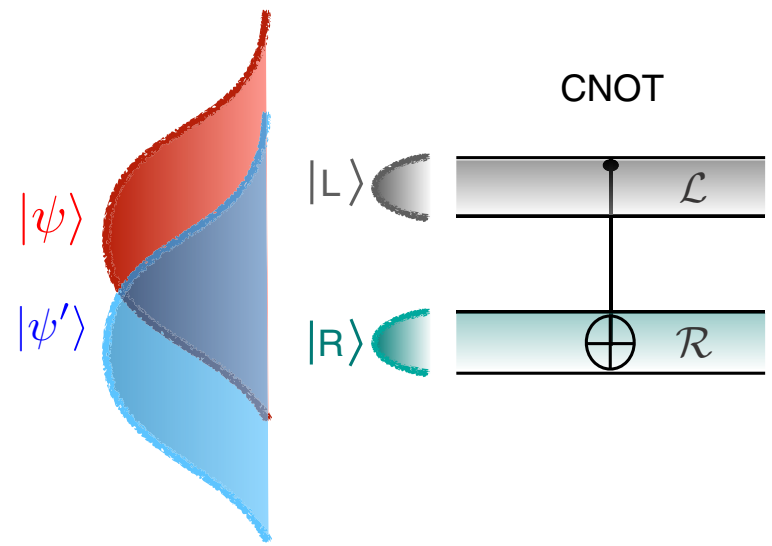

FIG. 1. CNOT gate for identical particles in the sLOCC framework.

where $\mathcal{N}$ is a global normalization factor, $l=\langle L \mid \psi\rangle, r^{\prime}=$ $\left\langle R \mid \psi^{\prime}\right\rangle, l^{\prime}=\left\langle L \mid \psi^{\prime}\right\rangle$, and $r=\langle R \mid \psi\rangle$ are the probability amplitudes of finding one particle in the two spatially separate states $|\mathrm{L}\rangle$ and $|\mathrm{R}\rangle$ and $\eta$ is 1 for bosons and -1 for fermions. Assuming, for instance, $l, r^{\prime} \neq 0$, the remaining probability amplitudes $l^{\prime}$ and $r$ are zero if and only if $|\psi\rangle$ and $\left|\psi^{\prime}\right\rangle$ do not spatially overlap. ${ }^{1}$ If $p_{\downarrow \uparrow}=p_{\uparrow \downarrow}=0$, the state $\rho^{\mathcal{I}}$ does not contain coherence, regardless of the identity of particles. Otherwise, the state is coherent if and only if the particles spatially overlap (are spatially indistinguishable). If they do not overlap, the state is incoherent: nonoverlapping identical particles act like nonidentical ones.

In the sLOCC framework, incoherent operations, known for nonidentical particles, can be straightforwardly translated in the context of identical particle systems. Let us consider for example the CNOT gate. In the standard implementation with nonidentical particles, there are individually addressed control and target spins. By analogy, if we have identical particles, we can identify the measurement regions $\mathcal{L}$ and $\mathcal{R}$ as control and target regions, respectively (Fig. 1). Applying it to the state of Eq. (3) when it is incoherent, it can be easily shown that it gives us an incoherent state. Therefore the CNOT gate remains an incoherent operation, which transforms incoherent states of identical particles into incoherent states.

\section{PHASE DISCRIMINATION PROTOCOL BY PARTICLE INDISTINGUISHABILITY}

Identical particles have been used for purposes of quantum metrology by means of particle-number states [24]. Our purpose here is to exploit the contribution to coherence due to the spatial indistinguishability, so determining its operational role in quantum information processing. It is known that within the context of quantum metrology, quantum coherence of states of nonidentical particles is a resource for phase

\footnotetext{
${ }^{1}$ Notice that such a constraint in the definition of spatial overlap is made here for the sake of simplicity. Certainly, a general definition of spatial overlap can be straightforwardly achieved by using projectors onto all the possible bound states in each region $\mathcal{L}$ and $\mathcal{R}$, which will be reported elsewhere.
} 


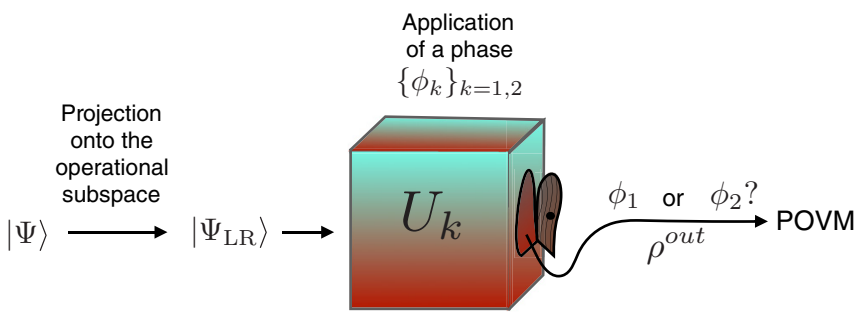

FIG. 2. Phase discrimination game.

discrimination tasks $[3,25]$. We now describe an analogous game for indistinguishability-enabled quantum coherence.

The state of two independently prepared nonidentical spins, for instance, $|\Psi\rangle_{\mathrm{AB}}=\left|\psi \downarrow_{\mathrm{A}}, \psi^{\prime} \uparrow_{\mathrm{B}}\right\rangle=|\psi \downarrow\rangle_{\mathrm{A}} \otimes$ $\left|\psi^{\prime} \uparrow\right\rangle_{\mathrm{B}}$, is manifestly incoherent in the basis $\mathcal{B}$. Differently, let us consider the corresponding pure state for two independently prepared identical spins

$$
|\Psi\rangle=\left|\psi \downarrow, \psi^{\prime} \uparrow\right\rangle,
$$

whose projection in the operational subspace spanned by $\mathcal{B}^{\mathcal{I}}$ is

$$
\left|\Psi_{\mathrm{LR}}\right\rangle=\frac{1}{\mathcal{N}_{\mathrm{LR}}}\left(l r^{\prime}|\mathrm{L} \downarrow, \mathrm{R} \uparrow\rangle+\eta l^{\prime} r|\mathrm{~L} \uparrow, \mathrm{R} \downarrow\rangle\right),
$$

with the normalization constant $\mathcal{N}_{\mathrm{LR}}=\sqrt{\left|l r^{\prime}\right|^{2}+\left|l^{\prime} r\right|^{2}}$. Notice that the resource (coherent) state above is conditionally prepared depending on the spatial overlap of the particles [18]. A nonzero coherence for this state can only stem from indistinguishability $\left(l^{\prime} r \neq 0\right)$. Let us send $\left|\Psi_{\mathrm{LR}}\right\rangle$ in a box where a unitary transformation $U_{k}=e^{i \hat{G} \phi_{k}}(\hat{G}$ is the generator of the transformation) applies one of the phases $\left\{\phi_{k}\right\}_{k=1,2}$ with a probability $p_{k}$ to the state (see Fig. 2). The requirement is that $U_{k}$ is a free (incoherent) operation, so $\hat{G}=$ $\sum_{\sigma \tau} \omega_{\sigma \tau}|\mathrm{L} \sigma, \mathrm{R} \tau\rangle\langle\mathrm{L} \sigma, \mathrm{R} \tau|$. The action of the box is

$$
\begin{aligned}
\left|\Psi_{\mathrm{LR}}^{k}\right\rangle & =U_{k}\left|\Psi_{\mathrm{LR}}\right\rangle \\
& =\frac{1}{\mathcal{N}_{\mathrm{LR}}}\left[l r^{\prime} e^{i \omega_{\downarrow \uparrow} \phi_{k}}|\mathrm{~L} \downarrow, \mathrm{R} \uparrow\rangle+\eta l^{\prime} r e^{i \omega_{\uparrow \downarrow} \phi_{k}}|\mathrm{~L} \uparrow, \mathrm{R} \downarrow\rangle\right] .
\end{aligned}
$$

Due to our ignorance of which of the two phases has been applied, the state coming out of the box is in the following classical mixture:

$$
\rho^{\text {out }}=\sum_{k=1,2} p_{k}\left|\Psi_{\mathrm{LR}}^{k}\right\rangle\left\langle\Psi_{\mathrm{LR}}^{k}\right| .
$$

Physically, the effect of the box can be realized with $\hat{G}$ corresponding to the Hamiltonian of two independent spins subject to localized magnetic fields $\mathrm{B}_{\mathcal{L}}^{k}$ and $\mathrm{B}_{\mathcal{R}}^{k}$, which randomly occur with a probability $p_{k}$.

The objective now is to establish which phase has actually been applied. In other words, the phase discrimination game translates in a state discrimination one. The two states $\left|\Psi_{\mathrm{LR}}^{1}\right\rangle$ and $\left|\Psi_{\mathrm{LR}}^{2}\right\rangle$ are in general not orthogonal: in order to discriminate them, a positive operator-valued measure (POVM) $[26,27]$ has to be chosen. We look for a POVM described by a set of operators $\left\{\hat{\Pi}_{1}, \hat{\Pi}_{2}\right\}$, each of which is associated to an outcome of the measurement: if we obtain 1, associated to the operator $\hat{\Pi}_{1}$, we conclude that the state coming of the box is
$\left|\Psi_{\mathrm{LR}}^{1}\right\rangle$, if the result is 2 , associated to the operator $\hat{\Pi}_{2}$, the state is $\left|\Psi_{\mathrm{LR}}^{2}\right\rangle$. The optimal POVM can be obtained by minimizing the probability of making an error in the discrimination, that is the probability of obtaining the result associated to $\hat{\Pi}_{k}$ when the state was $\left|\Psi_{\mathrm{LR}}^{k^{\prime}}\right\rangle$ with $k^{\prime} \neq k$. Such a probability is $[26,28]$

$$
P_{\text {err }}=p_{1}\left\langle\Psi_{\mathrm{LR}}^{1}\left|\hat{\Pi}_{2}\right| \Psi_{\mathrm{LR}}^{1}\right\rangle+p_{2}\left\langle\Psi_{\mathrm{LR}}^{2}\left|\hat{\Pi}_{1}\right| \Psi_{\mathrm{LR}}^{2}\right\rangle .
$$

Using the property $\sum_{k} \hat{\Pi}_{k}=\mathbb{I}$ [27], we can rewrite Eq. (8) as follows:

$$
P_{\text {err }}=p_{1}-\operatorname{Tr}\left[\left(p_{1}\left|\Psi_{\mathrm{LR}}^{1}\right\rangle\left\langle\Psi_{\mathrm{LR}}^{1}\left|-p_{2}\right| \Psi_{\mathrm{LR}}^{2}\right\rangle\left\langle\Psi_{\mathrm{LR}}^{2}\right|\right) \hat{\Pi}_{1}\right] .
$$

The minimization of $P_{\text {err }}$ is obtained when $\hat{\Pi}_{1}$ is the projector onto the positive eigenvector of the operator $\Delta=$ $p_{1}\left|\Psi_{\mathrm{LR}}^{1}\right\rangle\left\langle\Psi_{\mathrm{LR}}^{1}\left|-p_{2}\right| \Psi_{\mathrm{LR}}^{2}\right\rangle\left\langle\Psi_{\mathrm{LR}}^{2}\right|$. Choosing a rotated orthonormal basis $|0\rangle$ and $|1\rangle$ (with $|0\rangle$ slicing in half the angle between $\left|\Psi_{\mathrm{LR}}^{1}\right\rangle$ and $\left.\left|\Psi_{\mathrm{LR}}^{2}\right\rangle\right)$, we can write

$$
\left|\Psi_{\mathrm{LR}}^{1}\right\rangle=\cos \theta|0\rangle+\sin \theta|1\rangle, \quad\left|\Psi_{\mathrm{LR}}^{2}\right\rangle=\cos \theta|0\rangle-\sin \theta|1\rangle .
$$

The positive eigenvalue of $\Delta$ and the associated eigenvector are, respectively,

$$
\begin{aligned}
\lambda_{+} & =\frac{1}{2}\left(p_{1}-p_{2}+\sqrt{1-4 p_{1} p_{2}\left|\left\langle\Psi_{\mathrm{LR}}^{1} \mid \Psi_{\mathrm{LR}}^{2}\right\rangle\right|^{2}},\right. \\
|+\rangle & =\frac{1}{\mathcal{N}_{+}} a\left(\left|\Psi_{\mathrm{LR}}^{1}\right\rangle+\left|\Psi_{\mathrm{LR}}^{2}\right\rangle\right)+b\left(\left|\Psi_{\mathrm{LR}}^{1}\right\rangle-\left|\Psi_{\mathrm{RL}}^{2}\right\rangle\right),
\end{aligned}
$$

where $\quad \mathcal{N}_{+}=\sqrt{\cos ^{2} \theta \sin ^{2} \theta+\left[\lambda_{+}\left(p_{1}-p_{2}\right) \cos ^{2} \theta\right]^{2}}$, $a=\sin (\theta) / 2$ and $b=\left[\lambda_{+}-\left(p_{1}-p_{2}\right) \cos ^{2} \theta\right] /(2 \sin \theta)$. The optimal POVM, dependent on the spatial overlap of the two spins, is therefore $\{|+\rangle\langle+|, \mathbb{I}-|+\rangle\langle+|\}$, which gives the probability of error

$$
\begin{aligned}
P_{\text {err }} & =\frac{1}{2}\left(1-\sqrt{1-\left.4 p_{1} p_{2}|| \Psi_{\mathrm{LR}}^{1}\left|\Psi_{\mathrm{LR}}^{2}\right\rangle\right|^{2}}\right) \\
& =\frac{1}{2}-\sqrt{\frac{1}{4}-p_{1} p_{2}\left|\frac{\left|l r^{\prime}\right|^{2} e^{i \omega_{\downarrow} \phi_{12}}+\left|l^{\prime} r\right|^{2} e^{i \omega_{\uparrow \downarrow} \phi_{12}}}{\mathcal{N}_{\mathrm{LR}}^{2}}\right|^{2}},
\end{aligned}
$$

where $\phi_{12}=\phi_{1}-\phi_{2}$. The probability of error depends on the indistinguishability $\left(l^{\prime} r\right)$ and, for the chosen initial state, is independent of the statistics of particles. Our approach also allows us to evidence the effects of particle statistics on the game efficiency starting from a different initial state (see Sec. III A).

Let us fix $|l|^{2}=\left|r^{\prime}\right|^{2}=1 / 2$ : when the two particles spatially overlap with $\left|l^{\prime}\right|^{2}=|r|^{2}=\frac{1}{2}$, Eq. (12) reduces to

$$
\tilde{P}_{\mathrm{err}}=\frac{1}{2}\left(1-\sqrt{1-2 p_{1} p_{2} \cos ^{2}\left[\left(\frac{\omega_{\downarrow \uparrow}-\omega_{\uparrow \downarrow}}{2}\right) \phi_{12}\right]}\right),
$$

which is zero when $\left(\frac{\omega_{\downarrow \uparrow}-\omega_{\uparrow \downarrow}}{2}\right) \phi_{12}=\pi / 2$. The behavior of $P_{\text {err }}$ is displayed in Fig. 3. In particular in Fig. 3(a) we show, as a function of the phase difference, $\tilde{P}_{\text {err }}$ (blue solid line) and the probability of error $P_{\text {err }}$ for spatially separated particles $\left(l^{\prime}, r=0\right.$, orange dashed line). This latter case is analogous to that of nonidentical particles. Among the two, $\tilde{P}_{\text {err }}$ is always 
(a)

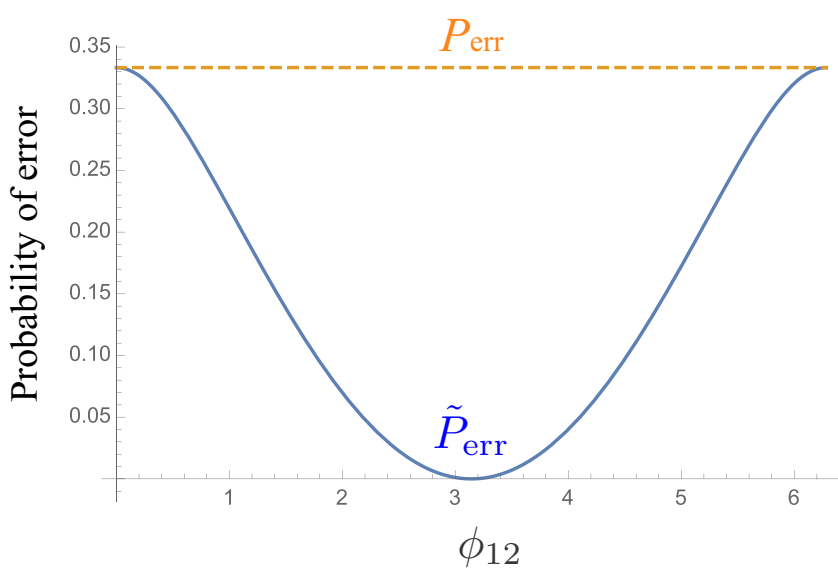

(b)

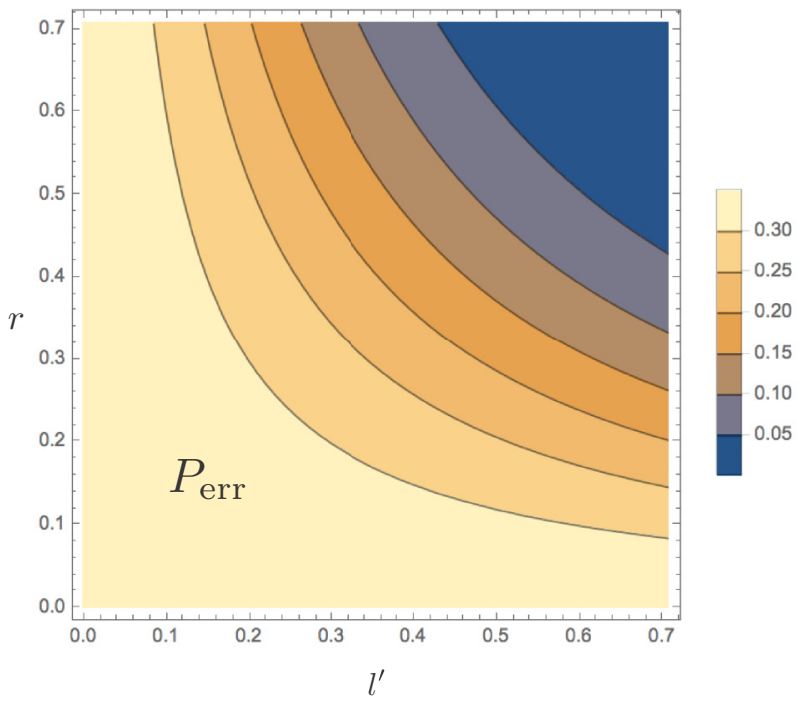

FIG. 3. (a) Probability of error in function of the phase difference $\phi_{12}$, with $p_{1}=1 / 3,|l|^{2}=\left|r^{\prime}\right|^{2}=\frac{1}{2}$, and $\omega_{\downarrow \uparrow}-\omega_{\uparrow \downarrow}=1$. The orange dashed line corresponds to $P_{\text {err }}$ [Eq. (12)] when there is no overlap $\left(l^{\prime} r=0\right)$ or, analogously, to the case with distinguishable particles. The blue one corresponds to $\tilde{P}_{\text {err }}$ [Eq. (13)], i.e., to the case of spatial overlap with $\left|l^{\prime}\right|^{2}=|r|^{2}=\frac{1}{2}$. (b) Contour plot of $P_{\text {err }}$ [Eq. (12)] in function of $r$ and $l^{\prime}$, with $\phi_{12}=\pi$.

smaller. For nonoverlapping spins, the state being incoherent, the best guess is to suppose that the most probable phase $\phi_{2}$ has been applied. The optimal probability of success is $P_{\text {succ }}=p_{2}$ and so $P_{\text {err }}=1-P_{\text {succ }}=p_{1}$ [see orange dashed line in Fig. 3(a)]. In Fig. 3(b) a contour plot of $P_{\text {err }}$ in terms of $l^{\prime}$ and $r$ is shown, in the case $\phi_{12}=\pi$. The optimal choice to minimize $P_{\text {err }}$ is to have two overlapping identical spins with $\left|l^{\prime}\right|^{2} \sim|r|^{2} \sim 1 / 2$.

\section{A. Role of particle statistics}

The phase discrimination game, within the state of Eq. (4), is independent of statistics. However, for a generic state this characteristic is not maintained. As an example, let us consider the following state:

$$
|\Phi\rangle=\frac{1}{\mathcal{N}^{\Phi}}\left|\psi \downarrow, \psi^{\prime} s\right\rangle,
$$

where $|s\rangle=a|\uparrow\rangle+b|\downarrow\rangle$, with $a, b \in \mathbb{R}$, and $\mathcal{N}^{\Phi}$ the normalization constant. Projecting $|\Phi\rangle$ in the operational subspace spanned by the identical basis $\mathcal{B}^{\mathcal{I}}=\{|\mathrm{L} \sigma, \mathrm{R} \tau\rangle, \sigma, \tau=\downarrow, \uparrow\}$, we obtain

$$
\left|\Phi_{\mathrm{LR}}\right\rangle=\frac{1}{\mathcal{N}_{\mathrm{LR}}^{\Phi}}\left(\left|\mathrm{L} \downarrow, \mathrm{R}^{\prime}\right\rangle+a \eta l^{\prime} r|\mathrm{~L} \uparrow, \mathrm{R} \downarrow\rangle\right),
$$

where $\quad\left|s^{\prime}\right\rangle=a l r^{\prime}|\uparrow\rangle+b\left(l r^{\prime}+\eta l^{\prime} r\right)|\downarrow\rangle, \quad \mathcal{N}_{\mathrm{LR}}^{\Phi}=$ $\sqrt{a^{2}\left(\left|l r^{\prime}\right|^{2}+\left|l^{\prime} r\right|^{2}\right)+b^{2}\left|l r^{\prime}+\eta l^{\prime} r\right|^{2}}$. The state of Eq. (14) is coherent in $\mathcal{B}^{\mathcal{I}}$ [see Eq. (15)] and in the chosen framework of spatially localized measurements, a contribution to the coherence due only to indistinguishability can be identified when particles spatially overlap $\left(l^{\prime} r \neq 0\right)$. The latter indistinguishability-enabled contribution plays a statistics-dependent operational role in a phase discrimination game. Let us send $\left|\Phi_{\mathrm{LR}}\right\rangle$ in the previously introduced box, in which a unitary transformation $U_{k}=e^{i \hat{G} \phi_{k}}$ applies one of the phases $\left\{\phi_{k}\right\}_{k=1,2}$ to the state with a probability $p_{k}$ and $\hat{G}$ is the generator of the transformation previously used. We thus obtain

$$
\begin{aligned}
\left|\Phi_{\mathrm{LR}}^{k}\right\rangle= & U_{k}\left|\Phi_{\mathrm{LR}}\right\rangle \\
= & \frac{1}{\mathcal{N}_{\mathrm{LR}}^{\Phi}}\left[a\left(l r^{\prime} e^{i \omega_{\downarrow \uparrow} \phi_{k}}|\mathrm{~L} \downarrow, \mathrm{R} \uparrow\rangle+\eta l^{\prime} r e^{i \omega_{\uparrow \downarrow} \phi_{k}}|\mathrm{~L} \uparrow, \mathrm{R} \downarrow\rangle\right)\right. \\
& \left.+b\left(l r^{\prime}+\eta l^{\prime} r\right) e^{i \omega_{\downarrow} \phi_{k}}|\mathrm{~L} \downarrow, \mathrm{R} \downarrow\rangle\right] .
\end{aligned}
$$

When the state comes out of the box, it is in a classical mixture

$$
\rho_{\Phi}^{\text {out }}=\sum_{k=1,2} p_{k}\left|\Phi_{\mathrm{LR}}^{k}\right\rangle\left\langle\Phi_{\mathrm{LR}}^{k}\right|,
$$

which represents our ignorance of which phase has been applied to the state. Using the first line of Eq. (12), in which $\left|\Psi_{\mathrm{LR}}^{i}\right\rangle$ is substituted by $\left|\Phi_{\mathrm{LR}}^{i}\right\rangle$, the probability of making an error in the discrimination game is now

$$
P_{\mathrm{err}}=\frac{1}{2}\left(1-\sqrt{1-4 p_{1} p_{2}\left|\frac{1}{\mathcal{N}_{R L}^{2}}\left[a^{2}\left(\left|l r^{\prime}\right|^{2} e^{i \omega_{\downarrow \uparrow} \phi_{12}}+\left|l^{\prime} r\right|^{2} e^{i \omega_{\uparrow \downarrow} \phi_{12}}\right)+b^{2}\left|l r^{\prime}+\eta l^{\prime} r\right|^{2} e^{i \omega_{\downarrow \downarrow} \phi_{12}}\right]\right|^{2}}\right),
$$

where $\phi_{12}=\phi_{1}-\phi_{2}$. 


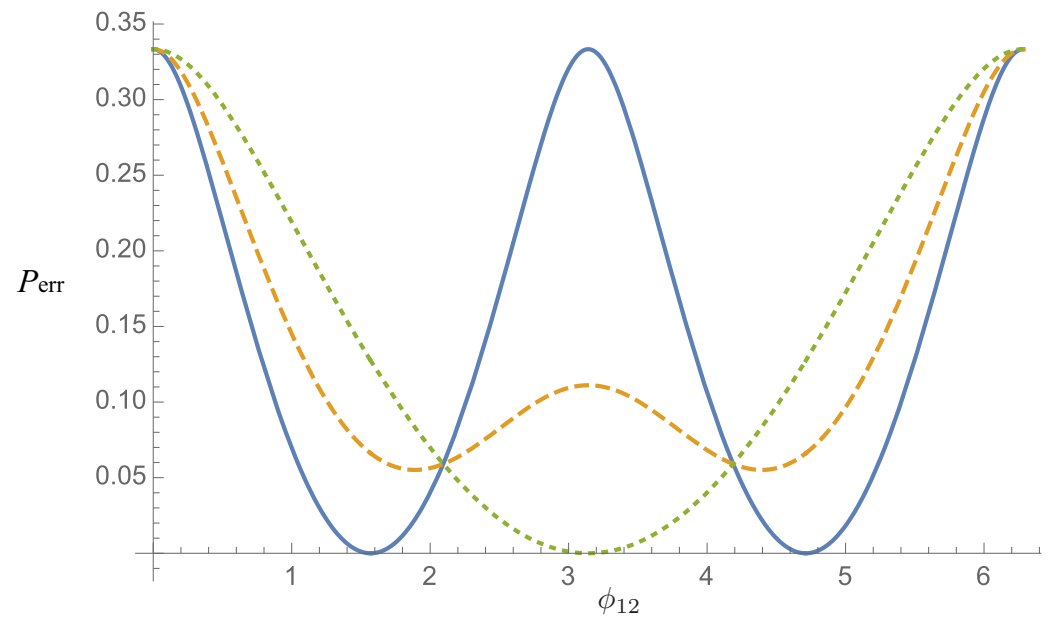

- = - Indistinguishable bosons

— Distinguishable particles

....... Indistinguishable fermions

FIG. 4. Probability of error in function of $\phi_{12}$ with $|l|^{2}=\left|r^{\prime}\right|^{2}=1 / 2$ for identical particles initially prepared in the state of Eq. (14): distinguishable (blue solid line) and indistinguishable with $\left|l^{\prime}\right|^{2}=|r|^{2}=1 / 2$ (orange-dashed line for bosons and green dotted line for fermions), with $a=b, \omega_{\downarrow \uparrow}=3, \omega_{\uparrow \downarrow}=2$ and $\omega_{\downarrow \downarrow}=1$.

From Figs. 4 and 5 it is easy to see that for certain ranges of the parameters $\phi_{12}$ and $\omega_{\sigma \tau}(\sigma, \tau=\downarrow, \uparrow)$, the probability of error using indistinguishable particles is considerably smaller than the one associated to distinguishable ones. In particular, in these ranges, fermions are more advantageous than bosons.

\section{CONCLUSIONS}

In this work we have defined the coherence for a system of two identical particles with two-level internal degrees of freedom in the framework of spatially local operations and classical communication (sLOCC). We have shown that the indistinguishability of identical particles is a source of coherence: while independently prepared distinguishable particles are incoherent under local operations, the analogous configuration with indistinguishable particles can exhibit quantum coherence. In our definition of coherence, one can naturally identify the contribution to coherence exclusively due to the spatial overlap of wave functions. In the sLOCC framework, the single- and two-particle incoherent operations known for distinguishable particles are straightforwardly generalized to systems of indistinguishable ones, as we have shown, for instance, in the case of CNOT gate.

We have then exploited the role of this indistinguishabilityenabled coherence in the context of quantum metrology. In particular, we have presented a phase discrimination protocol, for which we can explicitly demonstrate the operational advantage of using indistinguishable rather than distinguishable particles. Concretely, the coherence due to the quantum

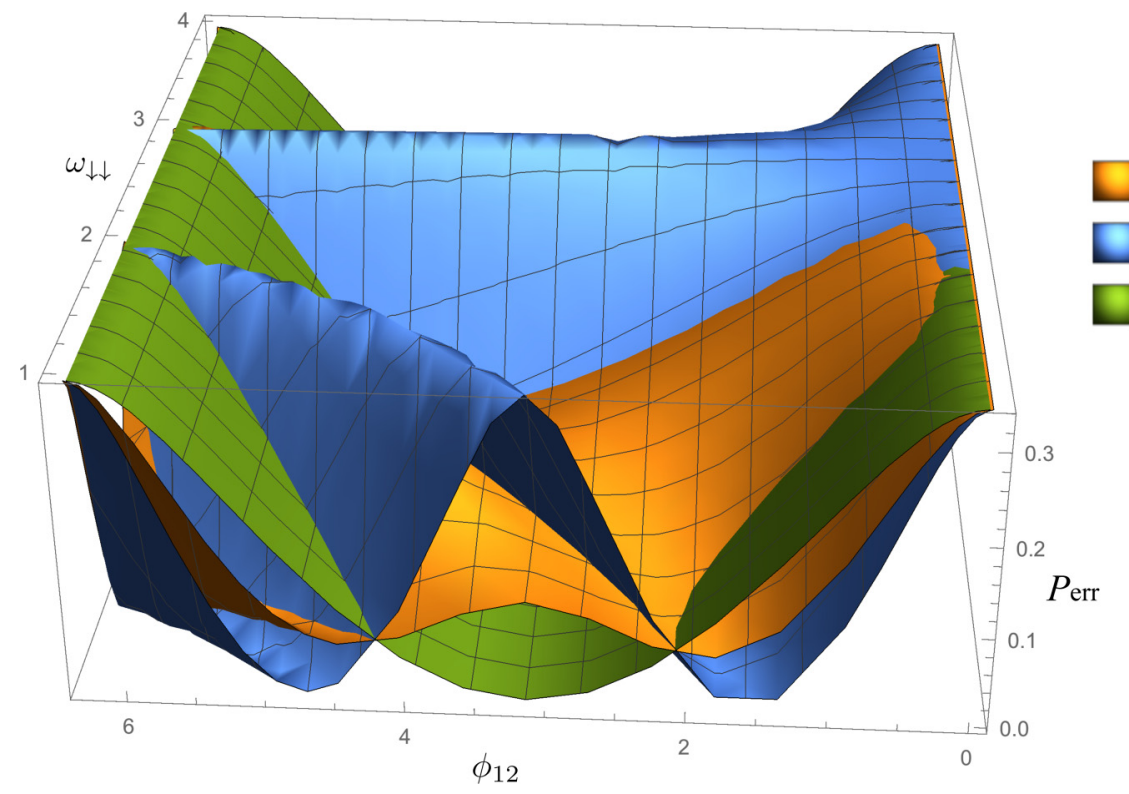

Indistinguishable bosons

Distinguishable particles

Indistinguishable fermions

FIG. 5. Three-dimensional (3D) plot of the probability of error with $|l|^{2}=\left|r^{\prime}\right|^{2}=1 / 2$ for identical particles initially prepared in the state of Eq. (14): distinguishable (blue) and indistinguishable with $\left|l^{\prime}\right|^{2}=|r|^{2}=1 / 2$ (orange for bosons and green for fermions) in function of $\phi_{12}$ and of $\omega_{\downarrow \downarrow}$, with $|l|^{2}=\left|r^{\prime}\right|^{2}=1 / 2, a=b, \omega_{\downarrow \uparrow}=3, \omega_{\uparrow \downarrow}=2$. 
indistinguishability significantly reduces the error probability of guessing the phase using the most general measurements. Moreover, we have shown how the particle statistics affects the coherence of a system of two identical spins and the efficiency of a phase-discrimination game.

The possibility of creating quantum coherence from a state of independently prepared identical particles can therefore be of experimental interest, since the relevant parameter to be controlled is the spatial overlap of the wave functions. This aspect may thus make all the procedure experimentally friendly. In fact, on the one hand it could facilitate the state preparation process and, on the other hand, avoids the typical state fragility linked to the quantum superpositions of composite systems [29,30].

Our results may raise the interest of researchers working on coherence in order to investigate and exploit further the notion of indistinguishability in this context. This work makes it clear that spatial overlap of identical particles plays a role not only in the context of entanglement $[11,12,18,31]$ but also for another fundamental feature of quantum systems, such as coherence, with an impact in any coherence-based quantum information processes.

\section{ACKNOWLEDGMENTS}

A.C. thanks G.A. for the hospitality at the University of Nottingham. A.W. was supported by the Spanish MINECO (project FIS2016-86681-P) with the support of FEDER funds, and the Generalitat de Catalunya (project 2017-SGR-1127). L.L. and G.A. acknowledge financial support from the European Research Council (ERC StG GQCOP, Grant Agreement No. 637352).
[1] E. Chitambar and G. Gour, Phys. Rev. Lett. 117, 030401 (2016).

[2] T. Baumgratz, M. Cramer, and M. B. Plenio, Phys. Rev. Lett. 113, 140401 (2014).

[3] C. Napoli, T. R. Bromley, M. Cianciaruso, M. Piani, N. Johnston, and G. Adesso, Phys. Rev. Lett. 116, 150502 (2016).

[4] M. Piani, M. Cianciaruso, T. R. Bromley, C. Napoli, N. Johnston, and G. Adesso, Phys. Rev. A 93, 042107 (2016).

[5] J. I. de Vicente and A. Streltsov, J. Phys. A: Math. Theor. 50, 045301 (2016).

[6] A. Winter and D. Yang, Phys. Rev. Lett. 116, 120404 (2016).

[7] A. Streltsov, G. Adesso, and M. B. Plenio, Rev. Mod. Phys. 89, 041003 (2017).

[8] D. P. Pires, I. A. Silva, E. R. de Azevedo, D. O. Soares-Pinto, and J. G. Filgueiras, Phys. Rev. A 98, 032101 (2018).

[9] K. Korzekwa, M. Lostaglio, J. Oppenheim, and D. Jennings, New J. Phys. 18, 023045 (2016).

[10] J. P. Santos, L. C. Céleri, G. T. Landi, and M. Paternostro, npj Quantum Inf. 5, 23 (2019).

[11] S. Bose and D. Home, Phys. Rev. Lett. 88, 050401 (2002).

[12] N. Paunković, Y. Omar, S. Bose, and V. Vedral, Phys. Rev. Lett. 88, 187903 (2002).

[13] S. Bose, A. Ekert, Y. Omar, N. Paunković, and V. Vedral, Phys. Rev. A 68, 052309 (2003).

[14] N. Killoran, M. Cramer, and M. B. Plenio, Phys. Rev. Lett. 112, 150501 (2014).

[15] Y. Omar, N. Paunković, S. Bose, and V. Vedral, Phys. Rev. A 65, 062305 (2002).

[16] Y. Omar, Int. J. Quantum Inf. 3, 201 (2005).
[17] A. Castellini, B. Bellomo, G. Compagno, and R. Lo Franco, Phys. Rev. A 99, 062322 (2019).

[18] R. Lo Franco and G. Compagno, Phys. Rev. Lett. 120, 240403 (2018).

[19] R. Lo Franco and G. Compagno, Sci. Rep. 6, 20603 (2016).

[20] G. Compagno, A. Castellini, and R. Lo Franco, Phil. Trans. R. Soc. A 376, 20170317 (2018).

[21] A. C. Lourenço, T. Debarba, and E. I. Duzzioni, Phys. Rev. A 99, 012341 (2019).

[22] G. C. Ghirardi, L. Marinatto, and T. Weber, J. Stat. Phys. 108, 49 (2002).

[23] M. C. Tichy, F. de Melo, M. Kus, F. Mintert, and A. Buchleitner, Fortschr. Phys. 61, 225 (2013).

[24] D. Braun, G. Adesso, F. Benatti, R. Floreanini, U. Marzolino, M. W. Mitchell, and S. Pirandola, Rev. Mod. Phys. 90, 035006 (2018).

[25] M. Ringbauer, T. R. Bromley, M. Cianciaruso, L. Lami, W. Y. Sarah Lau, G. Adesso, A. G. White, A. Fedrizzi, and M. Piani, Phys. Rev. X 8, 041007 (2018).

[26] C. W. Helstrom, Quantum Detection and Estimation Theory (Academic Press, New York, 1976).

[27] J. Audretsch, Entangled Systems (Wiley-VCH, Bonn, 2007).

[28] S. M. Barnett and S. Croke, Adv. Opt. Photon. 1, 238 (2009).

[29] W. H. Zurek, Nat. Phys. 5, 181 (2009).

[30] A. Perez-Leija, D. Guzmán-Silva, R. de J. León-Montiel, M. Gräfe, M. Heinrich, H. Moya-Cessa, K. Busch, and A. Szameit, npj Quantum Inf. 4, 45 (2018).

[31] S. Chin and J. Huh, Phys. Rev. A 99, 052345 (2019). 